\title{
Untersuchung des Wärmeübergangs von zyklisch beanspruchten Betonzylindern
}

\author{
Albert Vogel, Alexander Benz, Conrad Völker
}

\section{Copyright-Vermerk}

Copyright 2020 Ernst \& Sohn. Dieser Artikel kann für den persönlichen Gebrauch heruntergeladen werden. Andere Verwendungen bedürfen der vorherigen Zustimmung der Autoren und des Verlags Ernst \& Sohn.

Der folgende Artikel erschien in der Bauphysik 42(3), 131-138 (2020).

\begin{abstract}
Wiederkehrende Belastungen, wie sie beispielsweise an Brücken oder Windenergieanlagen auftreten, können innerhalb der Nutzungsdauer solcher Bauwerke bis zu $10^{9}$ Lastwechsel erreichen. Um das dadurch eintretende Ermüdungsverhalten von Beton zu untersuchen, werden diese zyklischen Beanspruchungen in mechanischen Versuchen mit Prüfzylindern nachgestellt. Damit Versuche mit solch hohen Lastwechselzahlen in akzeptablen Zeitdauern durchgeführt werden können, wird die Belastungsfrequenz erhöht. Als Folge dieser erhöhten Belastungsfrequenz erwärmen sich allerdings die Betonprobekörper, was zu einem früheren, unrealistischen Versagenszeitpunkt führen kann, weshalb die Erwärmung begrenzt werden muss. Um die Wärmefreisetzung in der Probe zu untersuchen, wurden Versuche und Simulationen durchgeführt. Im Beitrag wird die analytische und messtechnische Analyse des Wärmeübergangs an erwärmten Betonzylindern vorgestellt. Resultierend daraus wird eine Möglichkeit zur Reduktion der Erwärmung an zyklisch beanspruchten Betonzylindern vorgestellt.

Periodic load cycles, such as those that occur on bridges or wind turbines, are of great importance for dynamic long-term considerations of concrete. Within the life span of such structures, up to $10^{9}$ load changes can be expected. To determine the fatigue strength, the resistance to periodic loads can be determined in a short time in laboratory tests by increasing the load frequency. As a result of this increased load frequency, however, the concrete test specimens heat up, which can lead to an earlier, unrealistic time of failure, which is why the heating must be limited. Therefore, tests and simulations were carried out to investigate the heat release in the sample. In this article, the analytical analysis and measurements of the heat release of heated concrete cylinders are presented. As a conclusion, a possibility of reducing the temperature of dynamically stressed concrete cylinders is introduced.
\end{abstract}

\section{Einleitung}

Zur Untersuchung der Dauerstandfestigkeit von Beton werden Betonprobekörper dynamischer Lastbeanspruchung ausgesetzt. Dabei treten in Abhängigkeit von der Maximalbelastung und der Belastungsfrequenz signifikante Erwärmungen der untersuchten Betonzylinder auf [1]. Im Rahmen der Untersuchungen von Bode et al. [2] wurde eine Probenerwärmung von bis zu $\Delta \mathrm{T}=30 \mathrm{~K}$ festgestellt. Von Elsmeier et al. [3] wird sogar eine Erwärmung des Probekörpers um $\Delta \mathrm{T}=50 \mathrm{~K}$ genannt. Als Folge einer solchen Erwärmung kann es zu inkorrekten Prüfergebnissen hinsichtlich der Dauerstandfestigkeit kommen

Um die Erwärmung im Zuge der dynamischen Druckversuche zu verringern, muss der Erwärmungsprozess während der Versuche bekannt sein. Untersuchungen $[1,2]$ haben gezeigt, dass die Erwärmung der Probekörper auf die Dissipationsenergie zurückgeführt werden kann. Dieser 131
Zusammenhang konnte durch den Vergleich von gemessener mit simulierter Wärmeentwicklung bestätigt werden. Die Berechnung der Wärmeentwicklung anhand numerischer Simulationen ist von den thermischen Materialkennwerten Wärmeleitfähigkeit $\lambda$, spezifischer Wärmekapazität c und Dichte $\rho$ des Betons abhängig. Des Weiteren hat auch der Wärmeübergang von Zylindermantel zur Umgebung maßgeblichen Einfluss auf die Erwärmung der Betonprobe.

Eine mögliche Vorgehensweise zur Beschreibung bzw. Ermittlung des Wärmeübergangs ist der Vergleich bzw. die Kalibrierung eines numerischen Modells (z.B. CFD) mit experimentell ermittelten Daten, wie es durch von der Haar [4] vorgenommen wurde. Die Kalibrierung des Finite-Elemente-Modells (FE-Modell) ergab einen Wärmeübergang am Zylindermantel von $\alpha=5,5 \frac{\mathrm{W}}{\mathrm{m}^{2} \cdot \mathrm{K}}$ (Beton - Luft) sowie $\alpha=155 \frac{\mathrm{w}}{\mathrm{m}^{2} \cdot \mathrm{K}}$ an den Kontaktflächen zur Prüfmaschine (Beton - Stahl). Eine weitere 
Möglichkeit ist die analytische Berechnung sowie Messung des radiativen und konvektiven Wärmeübergangs am Prüfkörper, die im Folgenden vorgestellt wird.

\section{Modellbildung}

Der Wärmeübergang von der Zylindermanteloberfläche zur Umgebung wird hier durch ein radiatives und ein konvektives Teilmodell beschrieben, anschließend wird eine Validierung mittels Referenzmessungen durchgeführt.

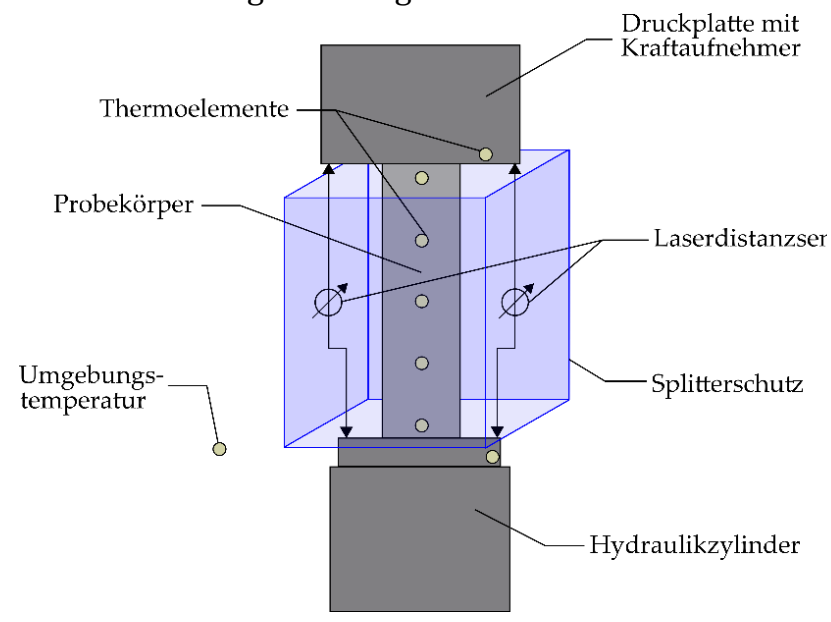

Bild 1. Betonprobekörper (Höhe: $300 \mathrm{~mm}$, Durchmesser: $100 \mathrm{~mm}$ ) in Prüfmaschine, umlaufender Splitterschutz aus Polymethylmethacrylat [2].

Fig. 1. Concrete cylinder (height: $300 \mathrm{~mm}$, diameter: 100 $\mathrm{mm}$ ) in testing machine, surrounding shatter protection made of polymethylmethacrylate [2].

Als Grundlage für die Modellbildung dienen die von Bode et al. [2] vorgestellten Belastungsversuche an Betonzylindern (Bild 1), die für unterschiedliche Belastungsfrequenzen und Oberspannungsniveaus durchgeführt wurden. Mithilfe dieser experimentellen Versuchsergebnisse wurden bereits numerische Modelle einschließlich des Wärmeübergangs kalibriert $[1,4]$. Im Folgenden wird dieser Kalibrierwert mit dem im vorliegenden Beitrag ermittelten Wärmeübergangskoeffizienten verglichen.

\subsection{Radiatives Teilmodell}

Der radiative Wärmeübergangswiderstand $\alpha_{r}$ wird durch den Strahlungsaustausch zwischen der Zylinderoberfläche und den Umgebungsflächen definiert. Die von der Zylinderoberfläche durch Strahlung an die Umgebung abgegebene Wärme ist dabei abhängig von der Größe der Umgebungsflächen und deren Oberfläche, vereinfacht zusammengefasst im Emissionskoeffizient $\varepsilon$. Im Zuge der Modellbildung wird der die Probe umgebende Splitterschutz vereinfachend über die Zylinderhöhe angenommen, das resultierende Strahlungsproblem lässt sich über eine thermische Ersatzschaltung lösen [5]. Dargestellt ist dieser Zusammenhang in Bild 2. Hierin können die Oberflächenwiderstände $\quad \mathrm{R}_{\mathrm{i}}=\frac{1-\varepsilon_{\mathrm{i}}}{\varepsilon_{\mathrm{i}} \cdot \mathrm{A}_{\mathrm{i}}} \quad$ sowie die Raumwiderstände $\quad \mathrm{R}_{\mathrm{ij}}=\frac{1}{\mathrm{~A}_{\mathrm{i}} \cdot \varphi_{\mathrm{ij}}} \quad$ für $\quad \mathrm{den}$ Strahlungsaustausch zwischen den Flächen $A_{i}$ und $A_{j}$ berechnet werden.

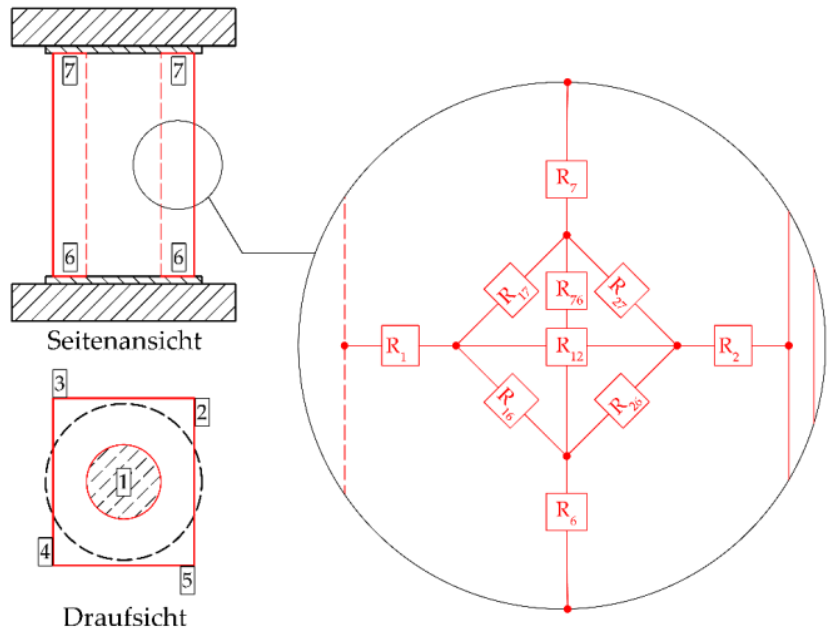

Bild 2. Strahlungsmodell und thermische Ersatzschaltung: Betonzylinder (1), Splitterschutz (2)-(5), Stahlflächen der Prüfmaschine (6), (7).

Fig. 2. Model for radiation and thermal equivalent network: concrete cylinder (1), shatter protection (2)-(5), steel surface of testing machine (6), (7).

Der Widerstand berechnet sich ausgehend von Fläche 1 folglich aus 6 Strahlungspfaden, siehe Gleichung (1). Die für weitere Berechnungen benötigten Einstrahlzahlen $\varphi_{\mathrm{ij}}$ sind über eine entsprechende Software berechnet worden [6]. Nach Gleichung (2) können die Strahlungskonstanten $\sigma_{\mathrm{ij}}$ der Flächenkombinationen (Strahlung von Fläche i zu Fläche j; $\sigma$ - Boltzmannkonstante) errechnet werden. Im weiteren Verlauf lassen sich die radiativen Wärmeübergangskoeffizienten $\alpha_{\mathrm{r}, \mathrm{ij}}$ (Gleichung (3)) beschreiben.

$$
\begin{aligned}
& \mathrm{R}_{\text {ges }}=\mathrm{R}_{1}+\frac{1}{\left(\frac{1}{\mathrm{R}_{12}+\mathrm{R}_{2}}+\cdots+\frac{1}{\mathrm{R}_{17}+\mathrm{R}_{7}}\right)} \\
& \sigma_{\mathrm{ij}}=\frac{1}{\frac{1-\varepsilon_{\mathrm{i}}}{\varepsilon_{\mathrm{i}}}+\frac{1}{\varphi_{\mathrm{ij}}}+\frac{1-\varepsilon_{\mathrm{j}}}{\varepsilon_{\mathrm{j}}} \cdot \frac{\mathrm{A}_{\mathrm{i}}}{\mathrm{A}_{\mathrm{j}}}} \sigma \\
& \alpha_{\mathrm{r}, \mathrm{ij}}=\sigma_{\mathrm{ij}} \cdot\left(\mathrm{T}_{\mathrm{i}}+\mathrm{T}_{\mathrm{j}}\right)\left(\mathrm{T}_{\mathrm{i}}^{2}+\mathrm{T}_{\mathrm{j}}^{2}\right)
\end{aligned}
$$


$\mathrm{T}_{i / j}$ ist hierbei die absolute Temperatur der Fläche $A_{i}$ bzw. $A_{j}$. Über eine Aufsummierung der Wärmeübergangskoeffizienten unter Berücksichtigung des jeweiligen Anteils am Strahlungsnetzwerk ergibt sich der Wärmeübergangskoeffizient $\alpha_{\mathrm{r}}$ (siehe Gleichung (4)).

$$
\alpha_{r}=\sum_{j=2}^{N}\left(\frac{R_{1+1 j+j}}{R_{\text {ges }}} \cdot \alpha_{1 j}\right)
$$

\subsection{Konvektives Teilmodell}

Der konvektive Wärmeübergang von Oberflächen zur angrenzenden Luftschicht ist von der Oberflächentemperatur abhängig. Die erwärmte Oberfläche bewirkt eine Erwärmung der Luft im Grenzschichtbereich und somit eine von der Probenhöhe abhängige nach oben gerichtete Konvektionsströmung. Für die Beschreibung des konvektiven Wärmeübergangs $\alpha_{c}$ sind mit der freien Konvektion und der Mischkonvektion zunächst zwei unterschiedliche Konvektionsbereiche definiert, wie in Bild 3 dargestellt.
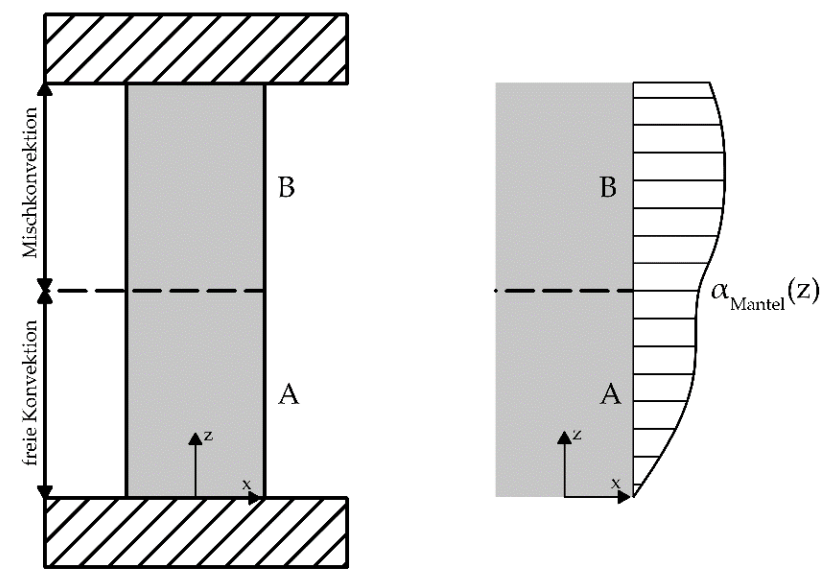

Bild 3. Bereichsdefinition für das Konvektionsmodell (links), qualitativer Verlauf des Wärmeübergangskoeffizienten $\alpha_{\text {Mantel }}(z)$ am Zylindermantel (rechts).

Fig. 3. Definition of areas for model of convection (left), qualitative trend of heat transfer coefficient $\alpha_{\text {sufface }}(z)$ at the cylinder's surface (right).

Über die Ähnlichkeitstheorie [7, 8] kann sowohl die freie als auch die erzwungene Konvektion beschrieben werden. Die für dieses Teilmodell genutzten Ähnlichkeitszahlen sind in Tabelle 1 aufgeführt und erläutert.

\section{Messung des Wärmeübergangs und der Strömungsgeschwindigkeit im Modellversuch}

Die zur Beschreibung der Reynolds-Zahl notwendige Strömungsgeschwindigkeit $\mathrm{v}$ in Längsrichtung des Zylindermantels wurde in Abhängigkeit einer eingeführten Bezugstemperatur $\theta^{*}=\frac{\theta_{\text {oberfläche }}-\theta_{\text {Luft }}}{\theta_{\text {Grenzschicht }}}$ beschrieben .

Hieraus konnte die Abhängigkeit $\mathrm{v}=0,0279 \ln \left(\theta^{*}\right)+0,1529\left[\frac{\mathrm{m}}{\mathrm{s}}\right]$ ermittelt werden. Die Durchführung der Versuche zur Beschreibung der von der Oberflächentemperatur abhängigen Strömungsgeschwindigkeit erfolgte durch Messungen mittels Hitzdrahtanemometern. Dabei wurde der Betonzylinder auf $60^{\circ} \mathrm{C}$ erwärmt und zur Nachbildung der Prüfsituation zwischen zwei runden Stahlplatten fixiert, welche die Ankopplung der Zylinder an die Druckplatten der Prüfmaschine nachbilden. Das Anemometer wurde für die Messung tangential in das Konvektionsfeld des abkühlenden Betonzylinders eingebracht (Bild 4b). Eine testweise Messung mit radial zum Konvektionsfeld orientiertem Anemometer, dargestellt in Bild 4a, zeigt keinen plausiblen Verlauf der zeitabhängig gemessenen Strömungsgeschwindigkeit, die hier trotz abkühlender Oberfläche steigt. Dies ist dadurch begründet, dass sich der Hitzdraht und der Temperatursensor zur Temperaturkompensation des Anemometers während der Messung auf unterschiedlichen Temperaturniveaus befanden. Bei tangentialer Orientierung des Anemometers hingegen finden die Hitzdraht- und Temperaturkompensationsmessung in einer isothermen Ebene statt, was messtechnisch korrekt ist. Die Strömungsgeschwindigkeit im Grenzschichtbereich nimmt mit der Zeit und sinkender Temperatur ab.

Die vollständige Beschreibung des Wärmeübergangs am Zylindermantel erfolgt durch die Aufsummierung unter Berücksichtigung der jeweiligen Mantelflächenanteile, die sich hier für den Zylinder auf die relativen Bereichslängen vereinfacht (Gleichung (5)).

$$
\begin{aligned}
& \alpha_{\text {ges }}=\frac{A_{A}}{A_{\text {ges }}} \cdot\left(\alpha_{c, A}+\alpha_{r, A}\right)+\frac{A_{B}}{A_{\text {ges }}} \cdot\left(\alpha_{c, B}+\alpha_{r, B}\right) \\
& =\frac{L_{A} \cdot \alpha_{A}+L_{B} \cdot \alpha_{B}}{L_{\text {ges }}}
\end{aligned}
$$


Tabelle 1. Übersicht der genutzten Ähnlichkeitszahlen.

Table 1. Overview of the used Likeness-Numbers.

\begin{tabular}{|c|c|c|}
\hline Art & Mathematische Definition & Physikalische Bedeutung \\
\hline \multicolumn{3}{|c|}{ Bereich A und B - Beschreibung der freien Konvektion } \\
\hline Lokale Grashof-Zahl & $\mathrm{Gr}_{\mathrm{z}}=\frac{\mathrm{g} \cdot \beta_{\mathrm{P}}\left|\theta_{\mathrm{W}}-\theta_{\infty}\right| \cdot \mathrm{z}^{3}}{v^{2}}$ & $\frac{\text { Auftriebskräfte }}{\text { Zähigkeitskräfte }}$ \\
\hline Prandtl-Zahl & $\operatorname{Pr}=\frac{v}{\mathrm{a}} \approx 0,7$ & $\begin{array}{l}\text { Impulstransport durch Reibung } \\
\text { Wärmetransport durch Leitung }\end{array}$ \\
\hline $\begin{array}{l}\text { Lokale } \\
\text { Rayleigh-Zahl }\end{array}$ & $\mathrm{Ra}_{\mathrm{D}}=\mathrm{Gr} \cdot \mathrm{Pr}$ & $\begin{array}{l}\text { Abgeleitete Kennzahl zur Beschreibung des } \\
\text { übertragenen Wärmeanteils durch Leitung und } \\
\text { Konvektion }\end{array}$ \\
\hline $\begin{array}{l}\text { Durchschnittliche } \\
\text { Rayleigh-Zahl }\end{array}$ & $\mathrm{Ra}_{\mathrm{D}}=\frac{1}{\mathrm{~L}_{\mathrm{A}}} \int_{0}^{\mathrm{L}_{\mathrm{A}}} \mathrm{Ra}_{\mathrm{z}} \mathrm{dz}$ & \\
\hline $\begin{array}{l}\text { Durchschnittliche Nußelt-Zahl für } \\
\text { freie Konvektion }\end{array}$ & $\mathrm{Nu}_{\mathrm{D}, \text { frei }}=\mathrm{C}_{1} \cdot\left(\mathrm{Ra}_{\mathrm{D}} \cdot \frac{\mathrm{D}}{\mathrm{L}}\right)^{\mathrm{a}_{1}}$ & $\begin{array}{l}\frac{\text { Wärmestrom durch Konvektion }}{\text { Wärmestrom durch Leitung }} \\
\text { Mit: } \mathrm{C}_{1}=1,75 ; \mathrm{a}_{1}=0,18\end{array}$ \\
\hline \multicolumn{3}{|c|}{$\alpha$ freie Konvektion } \\
\hline $\begin{array}{l}\text { Wärmeübergangskoeffizient für } \\
\text { Bereich A }\end{array}$ & $\alpha_{c, A}=\frac{\lambda_{\text {Luft }} \cdot \mathrm{Nu}_{\mathrm{D}}}{\mathrm{L}_{\mathrm{A}}}$ & \\
\hline \multicolumn{3}{|c|}{ Bereich B - Beschreibung der Mischkonvektion } \\
\hline Reynolds-Zahl & $\operatorname{Re}=\frac{w \cdot L_{B}}{v}$ & $\frac{\text { Trägheitskräfte }}{\text { Zähigkeitskräfte }}$ \\
\hline $\begin{array}{l}\text { Nußelt-Zahl für erzwungene, } \\
\text { laminare Konvektion }\end{array}$ & $\mathrm{Nu}_{\mathrm{lam}}=0,664 \cdot \mathrm{Re}^{\frac{1}{2}} \cdot \operatorname{Pr}^{\frac{1}{3}}$ & \\
\hline $\begin{array}{l}\text { Nußelt-Zahl für erzwungene, } \\
\text { turbulente Konvektion }\end{array}$ & $\mathrm{Nu}_{\text {turb }}=\frac{0,037 \cdot \mathrm{Re}^{0,8} \cdot \operatorname{Pr}}{1+2,443 \cdot \mathrm{Re}^{-0,1} \cdot\left(\operatorname{Pr} \frac{2}{3}-1\right)}$ & \\
\hline $\begin{array}{l}\text { Durchschnittliche Nußelt-Zahl für } \\
\text { erzwungene Konvektion }\end{array}$ & 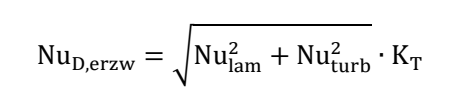 & $\begin{array}{l}\mathrm{K}_{\mathrm{T}}=\left(\frac{\mathrm{T}_{\mathrm{m}}}{\mathrm{T}_{\mathrm{W}}}\right)^{0,12} \text { als Korrekturfaktor für Gase für } \\
\text { die Temperaturabhängigkeit der Stoffwerte }\end{array}$ \\
\hline Nußelt-Zahl für Mischkonvektion & $N u_{\text {misch }}=\left(N u_{D, \text { erzw }}^{3}+N u_{D, \text { frei }}^{3}\right)^{\frac{1}{3}}$ & \\
\hline \multicolumn{3}{|c|}{$\alpha$ Mischkonvektion } \\
\hline $\begin{array}{l}\text { Wärmeübergangskoeffizient für } \\
\text { Bereich B }\end{array}$ & $\alpha_{c, B}=\frac{\lambda_{\text {Luft }} \cdot \mathrm{Nu}_{\text {misch }}}{L_{B}}$ & \\
\hline
\end{tabular}

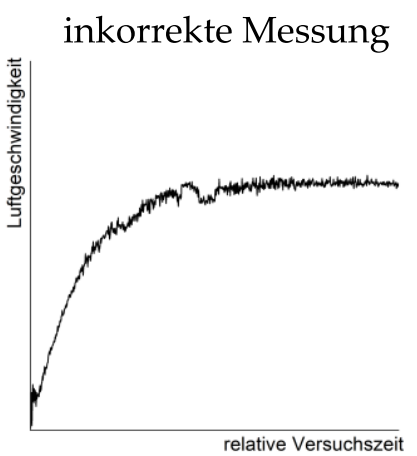

a)

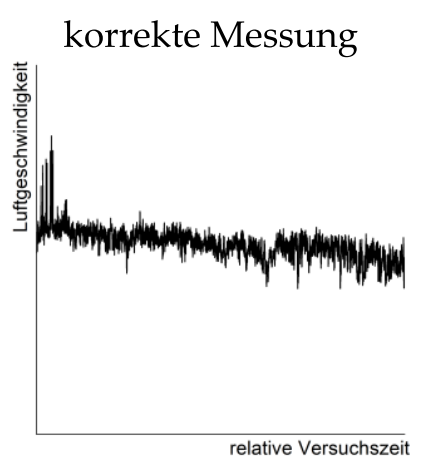

c)

Bild 4. Schematische Messanordnung (b) für die Strömungsmessung mit Hitzdrahtanemometer im Grenzschichtbereich des Betonprobekörpers; qualitative Verläufe der jeweils gemessenen Luftgeschwindigkeit: a) inkorrekte Lage des Anemometers; $b$ ) tangentiale Messanordnung

Fig. 4. Schematic measuring setup for flow measurement with hot wire anemometer in the boundary layer area of the concrete test specimen; qualitative curves of the air speed measured in each case: left - incorrect position of the anemometer; right - tangential measuring arrangement 
Bild 5 stellt den Verlauf des kombinierten Wärmeübergangskoeffizienten nach Gleichung (5) sowie die jeweiligen Anteile der Strahlung und Konvektion dar.

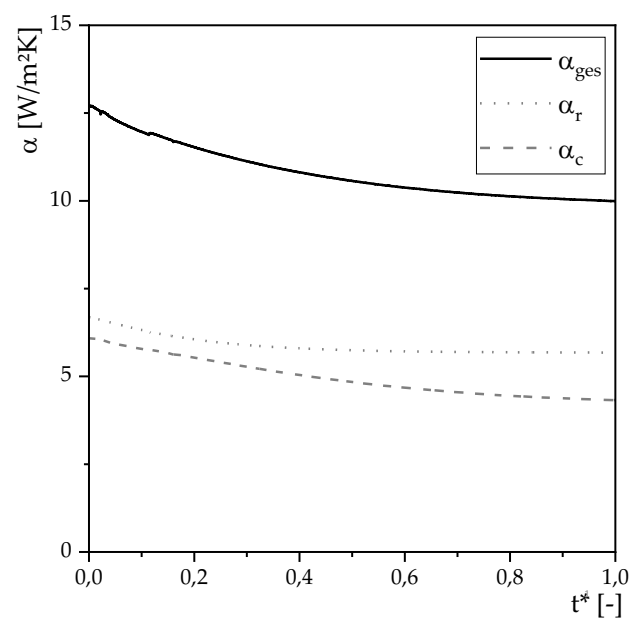

Bild 5. Verlauf des Wärmeübergangskoeffizienten $\alpha_{g e s}$ am Zylindermantel sowie die jeweiligen Strahlungsund Konvektionsanteile, dargestellt über die relative Versuchszeit $t^{*}$.

Fig. 5. Heat transfer coefficient $\alpha_{\text {total }}$ at the cylinder's surface as well as the respective radiative and convective components, visualized over the relative time $t^{*}$.

\section{Modellvalidierung}

Zur Überprüfung der zuvor beschriebenen Berechnung von $\alpha_{\text {ges }}$ wurde die Wärmestromdichte $\dot{q}$ bei anliegender Temperaturdifferenz $\Delta \theta$ in Versuchen, mit einem Aufbau analog zu Kapitel 3, gemessen. Zusätzlich wurden flexible Wärmestrommessplatten aus Silikon vertikal in der Zylindermitte fixiert, die Temperaturmessung erfolgte über NTC-Sensoren. Die so gemessene Wärmestromdichte wurde mit dem Ergebnis des Berechnungsmodells verglichen. Für die Modellrechnung findet die gemessene Oberflächentemperatur $\left(\theta_{\text {Mantel }}\right)$ sowie die gemessene Temperatur in der Grenzschicht ( $\left.\theta_{\text {Grenzschicht }}\right)$ Verwendung. Bild 1 zeigt den Vergleich der gemessenen Wärmestromdichten mit der Modellrechnung $\left(\dot{\mathrm{q}}_{\text {Rechnung }}=\alpha_{\text {ges }} \cdot\left(\theta_{\text {Mantel }}-\theta_{\text {Grenzschicht }}\right)\right) . \quad$ Die Darstellung erfolgt über die relative Versuchszeit t*. Es ergibt sich im arithmetischen Mittel eine Abweichung von ca. 7 \%. Das entwickelte Berechnungsmodell für den Wärmeübergangskoeffizienten an der Betonzylinderoberfläche wird somit als erfolgreich validiert angesehen.

\section{Transfer auf reale Belastungsversuche}

Das validierte Modell wird nun auf die realen Belastungsversuche übertragen. Für diesen Modelltransfer müssen die unterschiedlichen mechanischen
Randbedingungen

(Belastungsfrequenz

$f_{P}$, Oberspannungsniveau $\mathrm{S}_{\max }$ ) berücksichtigt werden. Bild 7a stellt den Verlauf des Wärmeübergangskoeffizienten aus der Modellrechnung sowie dessen Strahlungs- und Konvektionsanteile für einen Betonzylinder dar. Bild $7 \mathrm{~b}$ gibt eine Übersicht der untersuchten Betonzylinder.

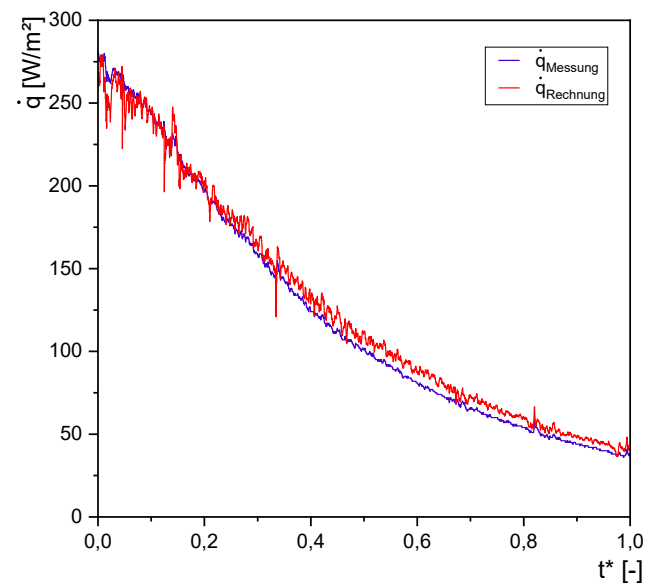

Bild 6. Vergleich der gemessenen und mit $\alpha_{\text {ges }}$ (Gleichung 5) berechneten Wärmestromdichte. Fig. 6. Comparison of the measured heat flux and those calculated with $\alpha_{\text {ges }}$ (equation 5).

Wie in Bild 7 dargestellt, nimmt der Wärmeübergangskoeffizient mit fortschreitender Versuchsdauer Werte zwischen 4,5 W/( $\left.\mathrm{m}^{2} \mathrm{~K}\right)$ und $5,5 \mathrm{~W} /\left(\mathrm{m}^{2} \mathrm{~K}\right)$ an. Dies unterstützt den durch von der Haar [4] mittels FEM-Kalibrierung ermittelten Wert für den Wärmeübergangskoeffizienten am Zylindermantel. Es ist anzumerken, dass für sehr kurze Belastungsversuche (Versuchsdauer $t_{\text {ges }}<20 \mathrm{~min}$ ) keine gesicherten Aussagen getroffen werden konnten. Es bedarf eines eingeschwungenen Zustands, der sich erst bei relativ langen Belastungszeiten einstellen würde.

\section{Parameterstudie zur Reduktion der Erwärmung}

Nach der erfolgreichen Validierung und Anwendung des Wärmeübergangsmodells auf die während der Belastungsversuche erwärmten Betonzylinder wurde nun eine Parameterstudie mit dem Simulationsprogramm ANSYS durchgeführt. Detaillierte Informationen zur Modellierung (Geometrie, Randbedingungen, Diskretisierung, physikalische Modelle etc.) können [1] entnommen werden. Ziel dieser Parameterstudie war die Minimierung sowohl der maximalen Temperatur als auch der maximalen Temperaturdifferenzen innerhalb des Probekörpers, da 


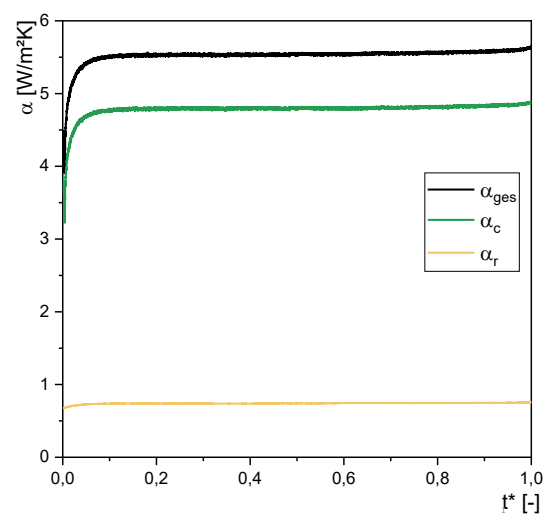

a)

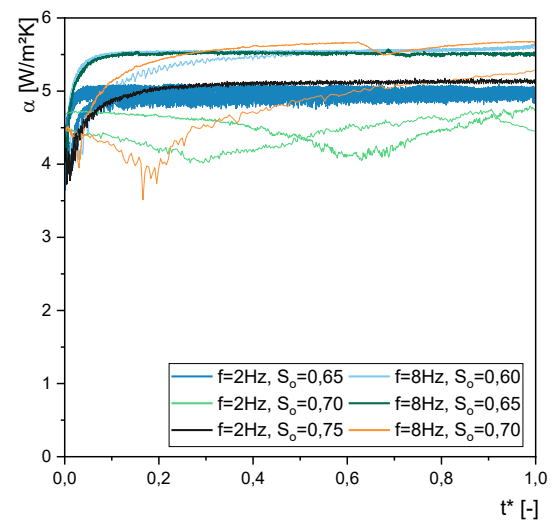

b)

Bild 7. a) Verlauf des Wärmeübergangskoeffizienten (für $f_{P}=8 \mathrm{~Hz}$, $S_{\max }=0,6$ ) sowie der zugehörigen Strahlungs- und Konvektionsanteile, b) Übersicht der Ergebnisse aus der Modellrechnung für die untersuchten Betonzylinder in Abhängigkeit der mechanischen Randbedingungen.

Fig. 7. a) Heat transfer coefficient (for $f_{P}=8 \mathrm{~Hz}, S_{\max }=0,6$ ) with the corresponding components of radiation and convection, b) summary of the results based on the developed model for the investigated specimens in dependence of the mechanical boundary conditions.

die dadurch auftretenden thermischen Spannungen das Probekörperversagen beeinflussen können. Aus diesem Grund wurden verschiedene Parameter, die die Temperaturentwicklung in der Betonprobe beeinflussen, im Rahmen der Simulationen untersucht. Hierzu zählten unter anderem die Materialparameter Rohdichte und Wärmeleitfähigkeit des Betons. Erwartungsgemäß zeigten die Simulationen, dass eine Erhöhung der Betonrohdichte sowie der Wärmeleitfähigkeit jeweils zu einer Verringerung der Probekörpertemperaturen führte, worauf im Folgenden allerdings nicht weiter eingegangen wird, da diese Parameter bei den dynamischen Belastungsversuchen entsprechend der Betonrezeptur konstant sind und nicht variiert werden können. Neben diesen Materialparametern wurde auch der Einfluss des Wärmeübergangs von der Zylindermantelfläche an die Umgebung im Rahmen der Parameterstudie untersucht. Aus diesem Grund wurden Simulationen mit Wärmeübergangskoeffizienten a Mantel in Höhe von 2, $5,10,20,50,75$ und $100 \mathrm{~W} /\left(\mathrm{m}^{2} \mathrm{~K}\right)$ durchgeführt. Ziel dieser Simulationen war es, die Möglichkeiten und Folgen eines erhöhten Wärmeübergangs durch Kühlung des Probekörpers zu eruieren

In Fehler! Verweisquelle konnte nicht gefunden werden. sind die Ergebnisse der Simulationen - hier die simulierten Maximaltemperaturen sowie der maximale Temperaturunterschied in der Probe in Abhängigkeit des Wärmeübergangskoeffizienten auf der Zylindermantelfläche - dargestellt. Daraus geht hervor, dass mit steigendem Wärmeübergang die maximale
Kerntemperatur sowie die maximale Temperaturdifferenz innerhalb des Probekörpers abnimmt. Weitere die Erwärmung beeinflussende Variablen sind die Anfangstemperatur der Probe, die Umgebungstemperatur sowie die Kühlung der Zylindergrundflächen (Kontaktflächen ProbePrüfmaschine). Die Variation dieser Umgebungsbedingungen bei den Simulationsrechnungen ergab zwar eine Reduktion der Maximaltemperaturen, allerdings keine wesentliche Änderung der Temperaturdifferenzen im Probekörper. Bei einer erhöhten Kühlung der Kontaktflächen zur Prüfmaschine kann sogar die maximale Temperaturdifferenz in der Probe steigen und dadurch zu erhöhten Temperaturspannungen führen, die wiederum $\mathrm{zu}$ einem vorzeitigen Versagen führen können. Die Umgebungstemperatur bzw. Temperatur der anströmenden Luft hat dabei ebenso Einfluss auf die absolute Maximaltemperatur und die Temperaturdifferenz im Probekörper, da bei niedriger Umgebungstemperatur mehr Wärme von der Zylinderoberfläche an die Umgebung abgegeben werden kann. Die Simulationsergebnisse zeigen eindeutig, dass durch den Wärmeübergang am Zylindermantel die größte Beeinflussung bzw. Reduktion der Probekörpertemperatur sowie der Temperaturdifferenz möglich ist. Es wird daher eine Mantelkühlung (z.B. Kühlmanschette oder erhöhte Luftkonvektion, siehe Folgeabschnitte) empfohlen. Oberflächenkühlung durch Kühlmanschette

Zur Untersuchung der Wirksamkeit einer Kühlmanschette wurden weitere ANSYS-Simulationen 
basierend auf den Daten eines dynamischen Druckversuches mit $f_{P}=10 \mathrm{~Hz}$ und $S_{\max }=0,6$ [4] durchgeführt, bei denen hohe Probenkörperkerntemperaturen von über $70{ }^{\circ} \mathrm{C}$ erreicht wurden.

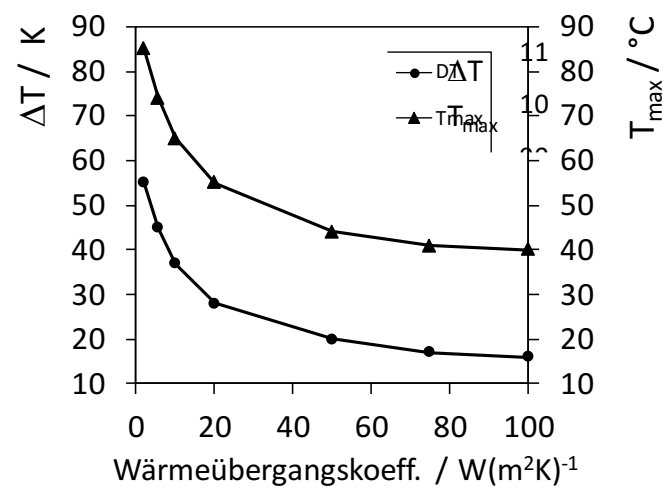

Bild 8. Maximaltemperatur und maximaler Temperaturunterschied im Probekörper in Abhängigkeit des Wärmeübergangskoeffizienten.

Fig. 8. Maximum temperature and maximum temperature-difference in the specimen in dependence of the heat transfer coefficient.

Die bei der Anwendung einer Kühlmanschette eigentlich durch Wärmeleitung abgeführte Wärme wurde in den Simulationen vereinfacht durch eine niedrige Umgebungstemperatur einschließlich verschiedener Wärmeübergangs-

koeffizienten $\alpha_{\text {Mantel }}$ simuliert. Da die Güte des wärmetechnisch soliden Kontaktes zwischen Kühlmanschette und Zylinderoberfläche nicht hinreichend genau bekannt ist, wurden verschiedene Werte für den Wärmeübergangskoeffizienten $\alpha_{\text {Mantel von }}$ $20-100 \mathrm{~W} / \mathrm{m}^{2} \mathrm{~K}$ gewählt. Dabei steht ein Wert von 100 $\mathrm{W} / \mathrm{m}^{2} \mathrm{~K}$ für einen sehr guten Verbund zwischen Kühlmanschette und Zylinderoberfläche. Bild 9 zeigt auszugsweise die Ergebnisse der Simulationen für Maximaltemperatur und Temperaturunterschied in Abhängigkeit des Wärmeübergangskoeffizienten am Zylinder-mantel bei Umschließung durch eine
Kühlmanschette mit einer am Mantel anliegenden Kühltemperatur von $0{ }^{\circ} \mathrm{C}$. Links in Bild 9 ist der Ausgangszustand ohne zusätzliche Kühlung dargestellt. Bei anliegender Kühlmanschette sinkt, in Abhängigkeit der Güte des Kontaktes zum Zylindermantel, die Probekörperkerntemperatur. Wird der Wärmeübergangskoeffizient $\alpha$ mantel ab $\mathrm{W} / \mathrm{m}^{2} \mathrm{~K}$ weiter erhöht, sinkt die Probekörpertemperatur nur noch leicht, während der maximale Temperaturunterschied sogar geringfügig zunehmen kann. Dieser Wert für aMantel ist somit für den untersuchten Fall als optimal hinsichtlich einer möglichst homogenen Temperaturverteilung bei gleichzeitiger geringer Maximaltemperatur im Probekörper anzusehen. Damit zeigt sich, dass der Einsatz einer Kühlmanschette bei optimierter Kühltemperatur bzw. Wärmeübergang durchaus sinnvoll sein kann, da durch die erzielte geringere Temperaturdifferenz im Probekörper auch die thermischen Spannungen verringert und dadurch der Versagensmechanismus beeinflusst wird. Die praktische Anwendung einer Oberflächenkühlung sowie die Auswirkungen der (reduzierten) inneren thermischen Spannungen auf das Versagen gilt es durch weitere Forschungsarbeit praktisch zu untersuchen.

\section{Oberflächenkühlung durch Konvektion}

Eine weitere Möglichkeit zur Erhöhung der Wärmeübergangskoeffizienten $\alpha$ Mantel ist die Queranströmung der Zylinder mit Raumluft. Die hierfür notwendige Anströmgeschwindigkeit quer zur Zylindermantelfläche ( $\left.v_{\text {quer}}\right)$ ergibt sich aus einem empirischen Ansatz, basierend auf Untersuchungen von Žukauskas [9] (siehe Gleichung (6)). Für den relevanten Bereich der durchgeführten Untersuchung ( $\alpha_{\text {Mantel }} 20$ $100 \mathrm{~W} / \mathrm{m}^{2} \mathrm{~K}$ ) ergibt sich der in Gleichung (6) dargestellte Zusammenhang für die mittlere NußeltZahl, wobei Pro die Prandtl-Zahl in Bezug auf die Zylindermanteltemperatur $\theta_{0}$ ist. Der radiative Wärmeübergang kann hierbei vernachlässigt werden,

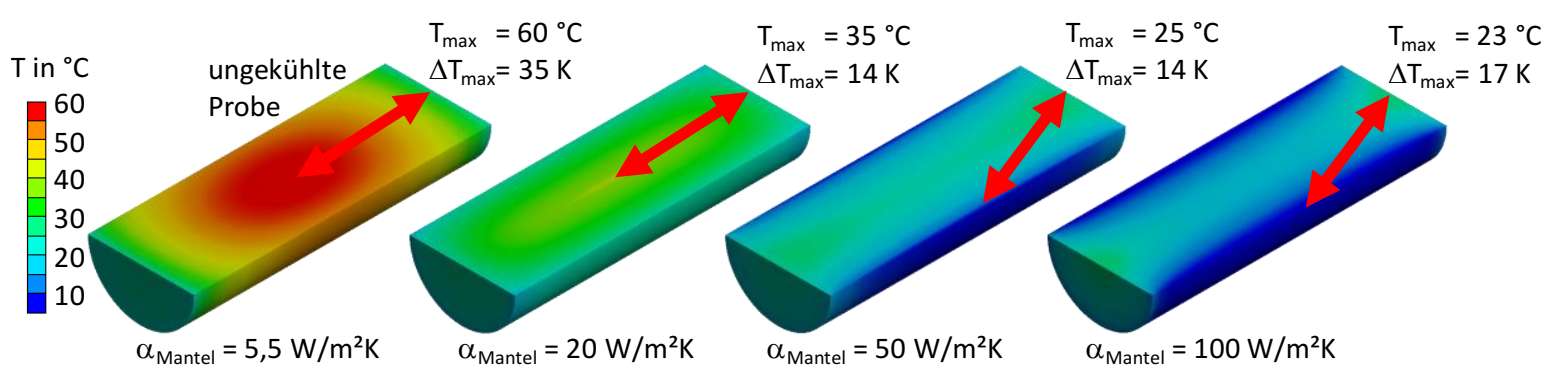

Bild 9. Simuliertes Temperaturfeld im Probekörper, maximale Temperaturdifferenz in Abhängigkeit des Wärmeübergangskoeffizienten an der Zylindermantelfläche; Kühltemperatur $0{ }^{\circ} \mathrm{C}$.

Fig. 9. Simulated temperature-distribution in the specimen, maximum difference in temperature in dependence of the heat transfer coefficient on the cylinder's surface; cooling temperature $0{ }^{\circ} \mathrm{C}$. 
da er bei einer solchen erzwungenen Konvektion eine untergeordnete Rolle spielt.

$$
\begin{aligned}
& \mathrm{Nu}\left(\mathrm{v}_{\text {quer }}\right)=0,26 \cdot \mathrm{Re}^{0,6} \cdot \operatorname{Pr}^{0,37} \cdot\left(\frac{\operatorname{Pr}}{\operatorname{Pr}_{0}}\right)^{0,25} \\
& \alpha_{\text {Mantel }}\left(\mathrm{v}_{\text {quer }}\right)=\frac{\lambda_{\text {Luft }} \cdot \mathrm{Nu}\left(\mathrm{v}_{\text {quer }}\right)}{\mathrm{L}_{\text {Zylinder }}}
\end{aligned}
$$

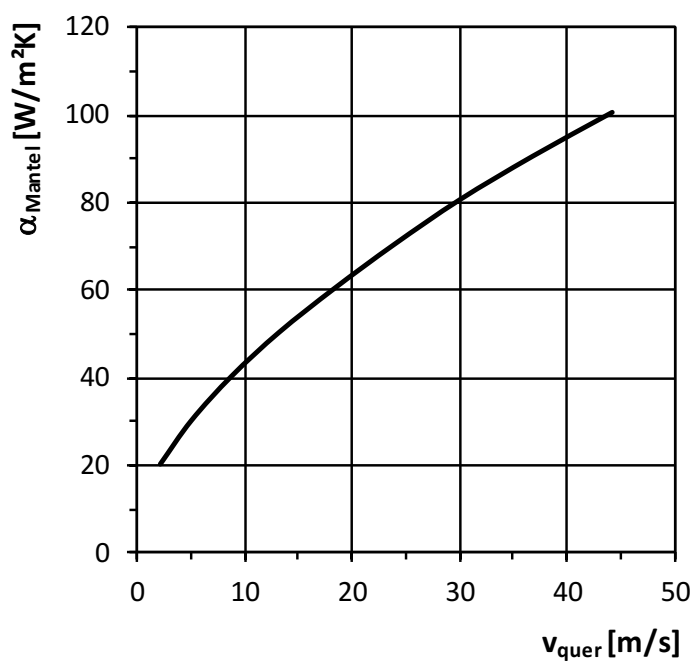

Bild 10. Verlauf des Wärmeübergangskoeffizienten $\alpha_{\text {Mantel }}$ in Abhängigkeit von der Anströmgeschwindigkeit $v_{\text {quer }}$ senkrecht zur Zylinderoberfläche (Lufttemperatur $20^{\circ} \mathrm{C}$ ).

Fig. 10. Surface heat transfer coefficient $\alpha_{\text {surface }}$ depending on the flow velocity $v_{\text {quer }}\left(v_{\text {crosswise }}\right)$ perpendicular to the cylinder's surface (air temperature $20^{\circ} \mathrm{C}$ ).

Bild 10 zeigt den Zusammenhang zwischen Anströmgeschwindigkeit vquer senkrecht zur Zylinderoberfläche und dem resultierenden Wärmeübergangskoeffizient, ohne Berücksichtigung der Konvektionsvorgänge längs des Zylindermantels. Zur Erreichung eines erhöhten Wärmeübergangs von $\alpha_{\text {Mantel }}=50 \frac{\mathrm{w}}{\mathrm{m}^{2} \mathrm{~K}}$ muss die technische Umsetzung zur Oberflächenkühlung somit einen erhöhten Luftstrom

\section{Literatur}

[1] Vogel, A., Völker, C., Bode, M., Marx, S.: Messung und Simulation der Erwärmung von ermüdungsbeanspruchten Betonprobekörpern. Bauphysik 42 (2020).

[2] Bode, M., Marx, S., Vogel, A., Völker, C.: Dissipationsenergie bei Ermüdungsversuchen an Betonprobekörpern. Beton- und Stahlbetonbau 114 (2019), S. 548-556.

[3] Elsmeier, K., Hümme, J., Oneschkow, N., Lohaus, L.: Prüftechnische Einflüsse auf das Ermüdungsverhalten hochfester feinkörniger Vergussbetone. Beton- und Stahlbetonbau 111 (2016), S. 233-240.

[4] Haar, C. von der, Wedel, F., Marx, S.: Numerical and Experimental Investigations of the Warming of FatigueLoaded Concrete: fib Symposium 2016. Kapstadt, Südafrika. mit $v_{\text {quer }}=15 \frac{\mathrm{m}}{\mathrm{s}}$ erzeugen können. Dies kann z.B. Zylinder umseitig mit 3 Gebläsen realisiert werden.

\section{7. $\quad$ Fazit}

Im Rahmen der durchgeführten Untersuchungen konnte ein Modell für den Wärmeübergang am Zylindermantel beschrieben und durch Laborversuche erfolgreich validiert werden. Des Weiteren konnte durch Auswertung von Belastungsversuchen die aus der Kalibrierung eines FE-Modells resultierenden Annahmen hinsichtlich des Wärmeübergangs im Wesentlichen bestätigt werden. Zudem besteht für die untersuchten Versuchsrandbedingungen die Möglichkeit der zeitabhängigen Beschreibung des Wärmeübergangskoeffizienten. Des Weiteren wurden Möglichkeiten zur Reduzierung der unerwünschten, übermäßigen Erwärmung der Betonproben untersucht. Dazu wurden Parameterstudien durchgeführt, deren Ergebnis die Empfehlung der Kühlung des Zylindermantels durch erzwungene Konvektion (Ventilatoren) oder durch eine Kühlmanschette ist. Dadurch kann die Probekörperkerntemperatur sowie der maximale Temperaturunterschied im Probekörper wesentlich gesenkt werden, was das frühzeitige Versagen der dynamisch beanspruchten Betonprobe verhindern soll.

\section{Danksagung}

Die Autoren bedanken sich bei der Deutschen Forschungsgemeinschaft für die Förderung des Projekts „Ursachen und Modellierung der Erwärmung von ermüdungsbeanspruchten Betonproben“ (DFGProjektnummer 342801069) sowie den Projektpartnern Prof. Dr.-Ing. Steffen Marx (TU Dresden) sowie Dipl.-Ing. Matthias Bode (Universität Hannover)

[5] Nitsche, K., Marek, R.: Praxis der Wärmeübertragung. Grundlagen, Anwendungen, Übungsaufgaben. München: Fachbuchverl. Leipzig im Carl Hanser Verl. 2007.

[6] Glück, B.: Software für numerische Berechnung von Einstrahlzahlen. http://berndglueck.de/waermeuebertragung.php, 10.03.2020.

[7] Baehr, H. D., Stephan, K.: Wärme- und Stoffübertragung. Berlin, Heidelberg: Springer-Verlag Berlin Heidelberg 2010.

[8] Nagendra, H. R., Tirunarayanan, M. A., Ramachandran, A.: Free Convection Heat Transfer From Vertical Cylinders Part I: Power Law Surface Temperature Variation. Nuclear Engineering and Design 16 (1970), S. 163-168.

[9] Žkauskas, A.: Heat Transfer from Tubes in Crossflow. Advances in Heat Transfer 18 (1987), S. 87-159. 